\title{
Beyond antisepsis: Examining the relevance of the works of Joseph Baron Lister to the contemporary surgeon-scientist
}

\author{
Graeme E. Glass \\ Nuffield Department of Orthopaedic, Rheumatology and Musculoskeletal Sciences, Kennedy Institute of Rheumatology, \\ University of Oxford, Oxford, OX3 7FY, United Kingdom
}

Address for correspondence: Dr. Graeme E. Glass, Nuffield Department of Orthopaedic, Rheumatology and Musculoskeletal Sciences, Kennedy Institute of Rheumatology, University of Oxford, Roosevelt Drive, Headington, Oxford, OX3 7FY, United Kingdom.

E-mail: graeme.glass@ndorms.ox.ac.uk

\section{ABSTRACT}

As the father of antispesis, the legacy of Joseph Baron Lister is assured and his influence on the development of contemporary surgical practice is recognised in the context of his achievement of predictable, infection-free surgery. However, looking beyond Lister's finest achievement and examining this work in the context of his whole career as a surgeon-scientist reveals important lessons pertinent to aspiring peers in how, by replacing surgical dogma with observation, deductive reasoning and scientific verification, by pursuing good ideas in the face of resistance and by making research directly relevant and patient-focused, lasting changes can be accomplished. This short paper aims to put Lister's developments in antisepsis in the context of his whole career, to evaluate his legacy and to commend his approach to contemporary surgeon-scientists.

\section{KEY WORDS}

Joseph Lister, surgery, antisepsis, inflammation, translational medicine

\section{INTRODUCTION}

n 36 BC Marcus Terentius Varro, Roman scholar, solider and prolific writer published his work entitled "On Agriculture" wherein he counselled against locating homesteads next to swap land because (in stagnant water) “...there are bred certain minute creatures that cannot be seen by the eyes, which float in the air and enter the body through the mouth and nose and there cause serious diseases." ${ }^{[1]}$

\begin{tabular}{|l|l|}
\hline \multicolumn{2}{|c|}{ Access this article online } \\
\hline Quick Response Code: & Website: \\
\hline & www.jps.org \\
\cline { 2 - 2 } & DoI: \\
\hline
\end{tabular}

In the West, this idea fell out of favour with the decline of the Roman Empire and it was not until the 1673 that Varro's prediction of "minute creatures" was proven by the Dutchman Antonie van Leeuwenhoek, whose pioneering work in microscopy made direct observation possible. Leeuwenhoek's letters were later published by the Royal Society in the journal "Philosophical Transactions" and are available as a web resource. ${ }^{[2]}$ The link between microscopic organisms and disease was later to be made by several authors. The Hungarian Obstetrician Ignaz Semmelweis noted the link between doctors who came into contact with puerperal fever and the deaths of subsequent patients from the same disease and advocated a hand washing regimen before and after patient contact. The English physician Dr. John Snow traced the 1854 outbreak of cholera to contaminated water by analysing the outbreak geographically. French chemist Louis Pasteur 
provided evidence for the refutation of the "miasma" theory of disease by demonstrating decomposition in the absence of air. He also linked bacterial virulence with disease and wound infection. ${ }^{[3]}$

Thus when, in 1867, the 40-year-old Joseph Lister, Professor of Surgery at the University of Glasgow published "on the antiseptic principle in the practice of surgery" in the British medical journal, the merits of the germ theory of disease were still being debated by the medical establishment. ${ }^{[4]}$ On his death some 45 years later, Lister's enduring legacy was to be known as the "father of antisepsis," having translated the germ theory of disease into a near universally adopted system of preventative surgery; an achievement for which he was awarded a Barony, fellowship of the Royal Society and membership of the Privy council. ${ }^{[5]}$ While it is for this reason that he is best remembered, Lister's researches focused on the interface between the experimental and the clinical and while his writings provide an illuminating insight into his many interests, the common thread running through his work (and the remarkable clarity of vision behind it) was the translational interface through which laboratory observation became meaningful clinical application. Moreover, Lister, like a number of his contemporaries, trialled a number of operations that are most certainly the domain of the plastic surgeon, including eyebrow reconstruction, cleft lip repair and skin grafting. ${ }^{[5]}$

\section{LISTER'S RESEARCHES}

As intimated in the $3^{\text {rd }}$ Huxley lecture, delivered before the medical school of Charing Cross hospital on October 2nd, 1900 , Lister's interest in the germ theory of disease as it related to surgery probably stemmed from his investigation into the death of a little boy on whom he had operated for a suppurating infection of the elbow while a resident house surgeon. ${ }^{[6]}$ Lister later summarised his theory in the opening passage of "on the antiseptic principle" thus: ${ }^{[4]}$

In the course of an extended investigation into the nature of inflammation and the healthy and morbid conditions of the blood in relation to it, I arrived ... at the conclusion that the ... cause of suppuration in wounds is decomposition brought about by the influence of the atmosphere upon blood or serum retained within them and in the case of contused wounds, upon portions of tissue destroyed by the violence of the injury. To prevent the occurrence of suppuration ... was an object manifestly desirable; but till late apparently unobtainable, since it seemed hopeless to exclude the oxygen, which was universally regarded as the agent by which putrefaction was effected. But when it had been shown by ... Pasteur that the septic property of the atmosphere depended ... on minute organisms suspended within it ... it occurred to me that decomposition of the injured part might be avoided without excluding the air, by applying as a dressing some material capable of destroying the life of the floating particles.

Lister advocated using carbolic or "phenic" acid (a potent antibacterial and antifungal agent) to kill contaminant flora and prevent further colonisation. In the paper entitled "illustrations of the antiseptic system of treatment in surgery" (Lancet 1867) Lister advocated carbolic acid dressings for incised wounds, open or lacerated wounds left to heal by secondary intention and drained abscesses. ${ }^{[7]} \mathrm{He}$ advocated either regular dressing changes or the addition of fresh carbolic acid to the dressings to ensure persistence of antisepsis. Lister modified his approach for cavitating wounds by advocating the use of a space-occupying paste of carbolic acid, carbonate of lime and linseed oil. Insightfully, he cautioned that the "reparative powers" of the tissues must be undisturbed. This concern led Lister to experiment with weaker concentrations of carbolic acid. Writing in the Lancet 1875 Lister abandoned the idea when he observed a rise in his infection rate..$^{[8]}$ In an address to the Glasgow Medico-Chirurgical society (later published in the BMJ 1868) Lister suggested changing only the top layers of a dressing and leaving the bottom layer intact..$^{\mid 9]}$ In another example of Lister's perspicacity, he experimented with rudimentary nonadherent dressings to make dressing changes more comfortable. ${ }^{[9]}$ These concerns strike a chord with contemporary surgeons who manage complex wounds as the importance of wound dressings, and the interface between dressing material and wound bed is still an area of intense investigation and speculation.

Lister's interest in preventing nosocomial infections led him to conclude that his antiseptic principles applied not only to theatre but to the wards as well. He highlighted the unhealthy atmosphere on hospital wards, suggesting that rather than a daily regimen of open windows (practiced in order to release miasma), judicious hand washing and instrumental sterilisation should be enforced. ${ }^{[10,11]}$ Recognising the role of haematoma in both infection and inflammation, Lister advocated the use of a rudimentary drainage system to avoid collections of blood, pus or 
serous fluid. ${ }^{[12]}$ In reconstructive surgery, drains remain a ubiquitous means of preventing haematoma and serous fluid accumulation.

It could be argued, from Lister's prodigious output on the subject, that inflammation remained Lister's primary scientific interest and that his interest in aseptic techniques stemmed for his pursuit of a means of moderating inflammation with the ensuing consequences of tissue necrosis and suppuration. In an address to the International Medical Congress of 1881, Lister cautioned that not all inflammation was due to the presence of bacteria and cautioned against the popular view that surgical debridement of all inflamed tissue was necessary or advantageous. ${ }^{[13]}$ Again, this view would find much support within the contemporary surgical community.

Lister experimented with chemical inflammation using the web space of a frog foot. He observed and documented changes in local capillary blood flow and permeability by the action of localised tissue insults such as mustard powder. ${ }^{[6]}$ Writing in "Philosophical Transactions" Lister reported his observation that localised inflammation caused the accumulation of "liquor sanguinis" (oedema) and red blood cell aggregation. ${ }^{[14]}$

In 1863 Lister presented the Croonian lecture to the Royal Society on the coagulation of blood. If some of these inquiries appear curiously misguided, it is only because our current understanding of vascular physiology supports such authority in retrospect. That said, Lister had observed that exposure of blood to foreign material resulted in clotting (preceding formal description of the intrinsic cascade ${ }^{[15]}$ and consequently cultivated the idea that inflammation of the lining of a vessel resulted in coagulation of the blood within it. He reasoned that the vascular occlusion increased pressure through a capillary bed leading to the formation of "liquor sanguinis" that further compromised localised tissue perfusion. ${ }^{[16]}$ Thus, Lister linked inflammation with tissue perfusion. In an address to the Harveian Society of London in 1878 Lister asserted that changes in blood vessel diameter as a result of position were not simply the result of hydrostatic principles but due to changes in the tension within the muscular vessel wall. ${ }^{[17]}$ He explained his theory thus.

"When a part has been deprived for a while of circulation, the want of the vital fluid creates in the tissues a demand for a supply of it, and that this demand operates on the vasomotor nervous apparatus of the $\operatorname{limb}$ as a stimulus inducing arterial relaxation."

Many years before a formal description of the regulatory systems on the coagulation cascade, Lister, before an audience of the Medical Society of London, described his observations of the capillary bed, suggesting that a regulatory anti-coagulatory system existed..$^{[14]}$

A meticulous observer, Lister also advocated using a microscope, (such as a compound microscope pioneered by his father,JosephJackson Lister) to examine pathological specimens. Listers' own scaled illustrations, published in the two-volume "collected papers" (Clarendon Press) in 1909, reveal his exactitude. ${ }^{[18]}$ Lister was to stress the need for accurate, scaled microscopic observations in his Huxley lecture of 1900.

In the field of experimental surgery, Lister was clearly interested in the reconstruction of form and function, leading him to design procedures for eyebrow reconstruction, cleft lip repair and skin grafting (both split and full-thickness) that he took from the inner arm. Notably, he may also have performed the first radical mastectomy (on his sister). He also described a method of closure of large open wounds by exploiting mechanical tissue creep. ${ }^{[5]}$ In the chapter entitled "On Amputation" for Holmes' System of Surgery, Lister advised that all amputations should be covered with a muscle flap, thus cushioning the stump and preventing skin breakdown. ${ }^{[19]}$ In another chapter, entitled "On Anaesthetics," Lister recommended pulling the tongue forward to maintain the airway when using chloroform general anaesthesia. He also proposed that the last meal should be excluded to prevent "troublesome vomiting during the inhalation."[20] These principles remain salient features of contemporary anaesthetic practice.

\section{LISTER'S LEGACY}

Thus, Lister published in the fields of physiology, pathology and clinical surgery and advanced the theory and practice of surgery in four key areas. These include the principle of antiseptic presurgical and surgical care, postsurgical wound management and ward practice; recognition of the roles of inflammation and tissue perfusion on wound healing and surgical wound management; microscopic analyses of specimens for pathological and microbiological diagnoses; and elegant 
reconstructive stratagems to meet the expectation of survival following major surgery.

Lister refused to accept that suppuration, sepsis and death were the anticipated sequelae of invasive surgery. His approach improved patient safety and focussed on outcomes, with infection-free survival being Lister's surgical goal. These attitudes, controversial at the time, are nothing less than the founding principles on which modern surgery is based. Thus, Lister's legacy is, in essence, a philosophical, as much as a practical one. By making surgical outcomes more predictable, Lister pioneered elective (and reconstructive) surgery as it is understood today. Importantly, the advances in surgical standards attributable to antiseptic regimens such as those of Lister and von Bergmann not only include contemporary aseptic protocols but rather, as a consequence of these principles, diseases and injuries that were considered inoperable on account of certainty of suppuration have become survivable. Hence, surgical techniques and interventions evolved to meet the expectation of survival. Lister was one such surgical pioneer, and the process gathered pace in the decades following acceptance of his antiseptic principles. For example, high energy lower limb trauma was (until Lister) usually fatal. Lister's own experience highlighted an example of one such injury, and the patient survived

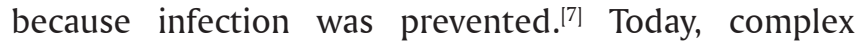
reconstructive options, including three-dimensional external fixation, vascularised tissue reconstruction of the soft tissue envelope and bone transport techniques have been developed and are widely practiced in order to meet the expected goal of infection-free fracture union. Infection-free wound closure is crucial to many other reconstructive stratagems.

Contemporary surgical antisepsis includes a presurgical washing regimen, formal antiseptic surgical preparation, surgery using sterilised instruments, postoperative dressings and wound management systems (including antibacterial dressings, drains and negative pressure wound therapy) designed to modulate the postoperative inflammatory response, prevent haematoma collection and promote infection-free wound healing. The growth of the dressings industry, with dressings tailored to facilitate healing of wounds of different sizes, depths and aetiologies, resonates with Lister's attempts to develop antibacterial and nonadherent dressings. Laminar flow theatre units have been developed to remove particulate debris from the atmosphere around the surgical site.
Given his writings on the subject, one feels that Lister would have been amused by the politicisation of hand washing protocols and the observance of hygiene protocols.

Lister recognised that haematoma resulted in localised tissue inflammation and served as a nidus for infection. The only means that Lister had at his disposal to prevent intra-operative bleeding and haematoma formation was drainage and surgical sutures, and indeed, Lister experimented with catgut and silver thread in an attempt to find alternatives to the braided silk that he recognized to be associated with infective complications. ${ }^{[6]}$ While the development of contemporary suture materials can be traced, in part, to Lister's quest for a safe and biologically inert suture, so too can the development of techniques designed to minimise intra-operative bleeding, including electrocautery, liga-clips, and alginate packs. To minimise intra-operative blood loss and improve postoperative healing Lister advocated techniques that are still in use today. In his address to the Harveian Society Lister proposed exanguination by elevation, followed by the application of a tourniquet, to achieve a bloodless field in hand and wrist surgery (a tourniquet had previously been used only for amputation). Moreover, Lister advocated postoperative splinting to improve pain and encourage healing. Lister's design for a hand and wrist splint achieves rest in the "position of function," favoured today. ${ }^{[21]}$

Lister's reliance on microscopy has served as a template for contemporary surgical diagnostic practice. Indeed, the integration of histology and microbiology as part of a multidisciplinary approach to surgical decision making is now considered an essential model for patient care. While Lister did not have antibiotics at his disposal he nevertheless aimed to identify the micro-organisms responsible for infections on the basis of their microscopic appearance. The principle of empirical antibiotic use, which would not be introduced until the 1960's, owes much to this approach.

\section{SUMMARY}

Lister's work has influenced many fields of surgery. However, when one reads Lister's papers, it is striking that of the many disparate topics that held his interest, antisepsis, inflammation and tissue perfusion, microbiology and reconstruction of form and function are threads that we, as plastic surgeons, may relate to. 
While most surgeons are likely to be aware of Lister's contribution to antisepsis, many may not be familiar with these additional interests. Nonetheless, we must not forget that, by striving for infection-free survival as a surgical imperative, Lister helped redefine surgical success and laid the foundations for the technical refinements and innovations to follow, including the evolution of new disciplines such as reconstructive and plastic surgery. With the advent of multi-drug resistance, the antiseptic principle in the practice of surgery promises to remain conceptually relevant today as it was in Lister's time. Arguably, the most important lesson that a surgeonscientist can learn by examining Lister's work is to challenge surgical dogma, resist skepticism and hostility to change, reflect on the translational implications of one's research output and, ultimately, to enhance patient experience.

\section{REFERENCES}

1. Cato MP, Varro MT. On Agriculture. Cambridge, MA: Loeb Classical Library, Harvard University Press; 1934. p. 576.

2. van Leeuwenhoek A. Collected Letters to the Royal Society. Available from: http://www.vanleeuwenhoek.com/Letters.htm. [Last accessed on 2013 Jun 24].

3. Pasteur L. Germ Theory and its Applications to Medicine and Surgery. Available from: http://www.fordhamedu/halsall/ mod/1878pasteur-germasp 1878. [Last accessed on 2014 Mar 24].

4. Lister J. On the antiseptic principle in the practice of surgery. $\mathrm{Br}$ Med J 1867;2:246-8.

5. Fisher RB. Joseph Lister 1827-1912. London: MacDonald and Jane's; 1977. p. 351.

6. Lister. The Huxley lecture. Br Med J 1900;2:969-77.
7. Lister J. Illustrations of the antiseptic system of treatment in surgery. Lancet 1867;2:668-9.

8. Lister J. On recent improvements in the details of antiseptic surgery. Lancet 1875;1:365-7.

9. Lister J. An address on the antiseptic system of treatment in surgery. Br Med J 1868;2:515-7.

10. Lister J. On the effects of the antiseptic system of treatment upon the salubrity of a surgical hospital. Lancet 1870;1:4-6.

11. Lister J. An address on the effect of the antiseptic treatment upon the general salubrity of surgical hospitals. $\mathrm{Br}$ Med $\mathrm{J}$ 1875;2:769-71.

12. Lister J. Clinical lecture on a case of excision of the knee joint, and horse hair as a drain for wounds. Lancet 1878;1:5-9.

13. Lister J. An Address on the Relations of Minute Organisms to Inflammation. Transactions of the International Medical Congress; 1881.

14. Lister J. On the coagulation of the blood in its practical aspects. Br Med J 1891;1:1057-60.

15. Lister J. On the coagulation of blood. Proc R Soc 1863.

16. Lister J. On spontaneous gangrene from arteritis and the causes of coagulation of the blood in diseases of the blood vessels. Edinb Med J, Apr. 1858.

17. Lister J. An address on the influence of position upon local circulation. Br Med J 1879;1:923-6.

18. Lister J. The Collected Papers of Joseph Baron Lister. Vol. 1. and 2. Clarendon Press, Oxford, UK: Clarendon Press; 1909.

19. Lister J. On amputation. In: Holmes T, Parckard JH, editors. Holmes' System of Surgery. Vol. 3. Philidelphia: H.C Lea's Son \& Co.; 1883.

20. Lister J. On anaesthetics. In: Holmes T, Parckard JH, editors. Holmes' System of Surgery. Vol. 3. Philidelphia: H.C. Lea's Son \& Co.; 1883.

21. Lister J. On examination of the wrist for caries. Lancet 1865;1:308-12.

How to cite this article: Glass GE. Beyond antisepsis: Examining the relevance of the works of Joseph Baron Lister to the contemporary surgeon-scientist. Indian J Plast Surg 2014;47:407-11.

Source of Support: Nil, Conflict of Interest: None declared. 